\title{
Tunneling-Injection Quantum-Dot Laser: Ultrahigh Temperature Stability
}

\author{
Levon V. Asryan and Serge Luryi, Fellow, IEEE
}

\begin{abstract}
We propose a genuinely temperature-insensitive quantum dot (QD) laser. Our approach is based on direct injection of carriers into the QDs, resulting in a strong depletion of minority carriers in the regions outside the QDs. Recombination in these regions, which is the dominant source of the temperature dependence, is thereby suppressed, raising the characteristic temperature $T_{0}$ above $1500 \mathrm{~K}$. Still further enhancement of $T_{0}$ results from the resonant nature of tunneling injection, which reduces the inhomogeneous line broadening by selectively cutting off the nonlasing QDs.
\end{abstract}

Index Terms-Quantum dots, quantum-well lasers, semiconductor heterojunctions, tunneling.

\section{INTRODUCTION}

$\mathbf{T}$ HE FIRST proposal of a semiconductor laser exploiting quantum dots (QDs) as the active medium was put forward by Arakawa and Sakaki in 1982 [1]. Ultrahigh temperature stability of operation was predicted to be one of the main advantages of QD lasers over conventional quantum-well (QW) lasers. Nevertheless, despite significant recent progress in the fabrication of QD lasers [2]-[11], their temperature stability has fallen far short of expectations. Even though the best results for the all-important parameter $T_{0}$, describing empirically the temperature dependence of the threshold current density $j_{\text {th }}$ and defined as $T_{0}=1 /\left(\partial \ln j_{\mathrm{th}} / \partial T\right)$, are quite respectable for QD lasers, matching, and even exceeding the best results reported for QW lasers at room temperature, so far they have been nowhere near the predicted "infinite" values that would allow one to regard the laser as temperature insensitive.

The dominant source of the temperature dependence of $j_{\text {th }}$ is parasitic recombination outside the QDs, primarily in the optical confinement layer $(\mathrm{OCL})$. In the conventional design, the OCL is a conductive medium where the QDs are embedded in such a way that carriers in the OCL and in QDs are in thermal equilibrium at room temperature. Consequently, the component of $j_{\text {th }}$ associated with recombination in the OCL depends exponentially on $T$ and the total threshold current becomes temperature dependent [12].

Another mechanism of the $T$-dependence in QD lasers is the inhomogeneous line broadening due to the QD size dispersion [13]. Experimental progress in controlling the QD parameters

Manuscript received January 23, 2001; revised March 28, 2001. This work was supported in part by the AFOSR MURI under Grant F49620-00-1-0331.

L. V. Asryan is with the State University of New York at Stony Brook, Stony Brook, NY 11794-2350 USA, on leave from the Ioffe Physico-Technical Institute, St. Petersburg 194021, Russia (e-mail: asryan@ece.sunysb.edu).

S. Luryi is with the State University of New York at Stony Brook, Stony Brook, NY 11794-2350 USA (e-mail: Serge.Luryi@ sunysb.edu).

Publisher Item Identifier S 0018-9197(01)05150-8. during the structure growth has been impressive; nevertheless, even in the best devices the measured gain and spontaneous emission spectra still indicate a significant QD size dispersion. Physically, the effect of QD size dispersion on $T_{0}$ is similar to that due to recombination in the OCL in the sense that the inhomogeneous line broadening is associated with undesired pumping of nonlasing QDs. So long as the electron and hole populations in the nonlasing QDs are in equilibrium with those in the active QDs, the fraction of $j_{\text {th }}$ arising from the recombination in nonlasing QDs depends on $T$ and the characteristic temperature $T_{0}$ is no longer infinite. Quantitatively, the effect of inhomogeneous broadening on $T_{0}$ is further discussed below.

Still another mechanism of the $T$-dependence of $j_{\text {th }}$ is associated with the violation of charge neutrality in QDs [14]. This leads to a temperature dependence of the recombination current in the lasing QDs themselves arising from the fact that carrier populations there are no longer fixed by the generation condition. Violation of charge neutrality is the dominant mechanism of temperature sensitivity at low temperatures [12] but is unimportant at $300 \mathrm{~K}$. In this article, we shall not concern ourselves with this effect and assume charge neutrality.

As will be shown below, elimination of the OCL recombination alone results in a dramatic improvement of the temperature stability. To accomplish this we propose a novel QD laser design, based on tunneling injection of carriers into the QDs wherein they recombine radiatively. Our design allows to both suppress the parasitic components of threshold current and diminish the effect of inhomogeneous line broadening.

Carrier injection by tunneling has been successfully tested in the context of QW lasers. Bhattacharya and coworkers [15]-[17] have realized tunneling-injection QW lasers and demonstrated improved modulation characteristics, lower wavelength chirp, and superior high-temperature performance as compared to conventional QW lasers.

\section{LASER STRUCTURE}

A schematic view of the structure and its energy band diagram are shown in Fig. 1. Basically, we have a separate confinement double-heterostructure laser. Electrons and holes are injected from $n$ - and $p$-cladding layers, respectively. The QD layer, located in the central part of the OCL, is clad on both sides by QWs separated from the QDs by thin barrier layers. Injection of carriers into QDs occurs by tunneling from the QWs. The key idea of the device is that the QWs themselves are not connected by a current path that bypasses the QDs. Electrons (coming from the left in Fig. 1) can approach the right QW only through the confined states in the QDs. Similarly, holes cannot directly approach the left QW. 


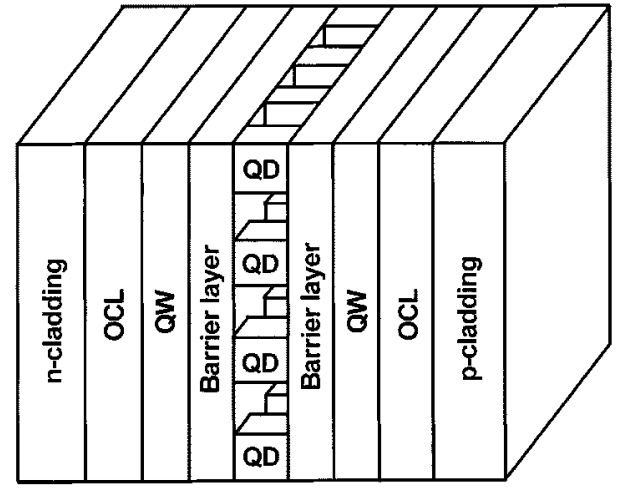

(a)

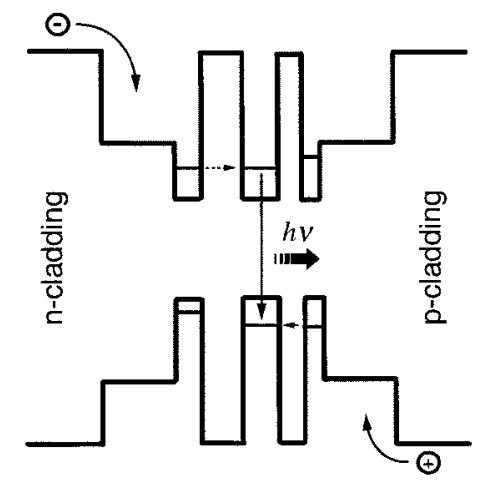

(b)

Fig. 1. (a) Schematic view and (b) the energy band diagram of a tunneling-injection QD laser. The QWs and the QDs (not drawn to scale) are assumed implemented in the same material, although this does not have to be necessarily the case in general. The electron-injecting QW is wider than the hole-injecting QW and both QWs are narrower than the QD to accomplish resonant alignment of the majority-carrier subbands with the QD energy levels. The tunnel barrier on the electron-injecting side is made thicker to suppress the leakage of the holes from the QD.

To realize this idea, the following conditions must be met.

1) The QW material and the thickness should be chosen so that the lowest subband edge in the injecting QW matches the quantized energy level for the corresponding type of carrier in the average-sized QD (the QWs may or may not be of the same material as the QDs).

2) The barriers should be reasonably high to suppress the thermal emission of carriers from the QWs.

3) The material separating QDs from each other in the QD layer should have a sufficiently wide bandgap to suppress all tunneling other than via the QD levels. This material may be the same as that of the barrier layers;

4) The barrier layers should be thin enough to ensure effective tunneling between the QW and QD states. At the same time, the separation between the adjacent QDs in the QD layer should be large enough to prevent any significant tunnel splitting of the energy levels in neighboring QDs (otherwise, such a splitting would effectively play the same role as the inhomogeneous line broadening).

We should note that a fraction of injected carriers might not recombine in the QD, but escape in a second tunneling step into the "foreign" QW and recombine with the majority carriers there. The size of this fraction depends on the ratio of the escape tunneling rate to the QD recombination rate and is practically independent of the temperature. Clearly, the escape tunneling does not lead to a temperature dependence of threshold current; nevertheless, in a sensible design, this form of leakage should be minimized to lower the value of $j_{\mathrm{th}}$. A possible way of suppressing the escape tunneling is illustrated in Fig. 1(b). It takes advantage of the lower electron effective mass compared to the hole mass, but uses this advantage differently on the electronand hole-injecting sides of the structure. On the p-side, when the hole level in the QD is aligned with the hole subband in the hole-injecting QW, the electron subband edge in that QW will be necessarily above the electron level in the QD, thus suppressing the tunneling escape of electrons. On the n-side, this trick does not work, since the resonant alignment of the electron subband in the QW and the electron level in the QD does not prevent tunneling of QD holes into the electron-injecting QW. However, due to the effective mass difference, we can design a wider tunnel barrier on the electron-injecting side, such that it effectively suppresses the tunneling escape of holes while still being relatively transparent for electrons.

\section{SUPPRESSION OF THE PARASITIC RECOMBINATION CHANNELS}

The general equation for the injection current density per unit area of the junction is

$$
\begin{aligned}
& j=e B^{3 \mathrm{D}} \int_{-b / 2}^{b / 2} n(x) p(x) d x+e B^{2 \mathrm{D}} \\
& \cdot \sum_{j=1}^{2} n_{j}^{2 \mathrm{D}} p_{j}^{2 \mathrm{D}}+\frac{e N_{\mathrm{S}}}{\tau_{\mathrm{QD}}}\left\langle f_{\mathrm{n}}(a) f_{\mathrm{p}}(a)\right\rangle
\end{aligned}
$$

where

$$
\begin{aligned}
& B^{3 \mathrm{D}} \quad \text { radiative constant for the OCL (given by [13, } \\
& n(x), p(x) \quad \text { densities of free electrons and holes in the } \\
& \text { OCL; } \\
& b \quad \text { OCL thickness; } \\
& B^{2 \mathrm{D}} \quad \text { radiative constant for the QWs; } \\
& n_{j}^{2 \mathrm{D}}, p_{j}^{2 \mathrm{D}} \quad \text { densities of 2-D electrons and holes in the two } \\
& \text { QWs }(j=1,2) \text {; } \\
& N_{\mathrm{S}} \quad \text { surface density of QDs; } \\
& \tau_{\mathrm{QD}} \quad \text { spontaneous radiative lifetime in QDs (given } \\
& \text { by [13, eq. (8)]); }
\end{aligned}
$$
ties in the QWs, and the confined-carrier level occupancies in the QDs are related by rate equations [13], [18].

The modal gain spectrum of a QD laser is given by

$$
\begin{aligned}
g(E)=\frac{8}{3} \pi^{2} \frac{\alpha}{\sqrt{\epsilon}} P^{2} & \frac{1}{E} \frac{1}{\frac{b}{2}+\frac{1}{\gamma}} N_{\mathrm{S}} \\
& \cdot\left\langle\left[f_{\mathbf{n}}(a)+f_{\mathbf{p}}(a)-1\right] \delta\left[E-E_{0}(a)\right]\right\rangle
\end{aligned}
$$


where

$\alpha \quad$ fine structure constant;

$\epsilon \quad$ dielectric constant of the OCL;

$P \quad$ Kane's parameter;

E photon energy;

$\gamma \quad$ decay constant of the electric field along the transverse direction in the cladding layers (to be found from the eigenvalue equation-see [13]);

$E_{0}(a)$ transition energy in a QD of size $a$.

The maximum (saturation) value of the modal gain spectrum peak is inversely proportional to the inhomogeneous line broadening $(\Delta \varepsilon)_{\text {inhom }}$

$$
g^{\max }=\frac{\xi}{4}\left(\frac{\bar{\lambda}_{0}}{\sqrt{\epsilon}}\right)^{2} \frac{1}{\tau_{\mathrm{QD}}} \frac{\hbar}{(\Delta \varepsilon)_{\text {inhom }}} \frac{1}{\frac{b}{2}+\frac{1}{\gamma}} N_{\mathrm{S}}
$$

where $\xi$ is a numerical constant $(\xi=1 / \pi$ and $\xi=1 / \sqrt{2 \pi}$ for the Lorentzian and Gaussian QD size distribution functions, respectively), $\bar{\lambda}_{0}=2 \pi \hbar c / \bar{E}_{0}$, and $\bar{E}_{0}=E_{0}(\bar{a})$ is the transition energy in the QD of the average size $\bar{a}$.

At lasing threshold, the peak spectral value of the modal gain equals the total loss $\beta$. This equation determines the values of the electron and hole level occupancies at the lasing threshold. (In the absence of charge neutrality, one needs another relation between the electron and hole level occupancies, which is derived self-consistently from electrostatic considerations). Using the evaluated level occupancies in the rate equations, one calculates $n, p$, and $n_{j}^{2 \mathrm{D}}, p_{j}^{2 \mathrm{D}}$, and eventually the threshold current density $j_{\text {th }}[$ see (1)].

In the proposed structure, carriers cannot bypass the QDs on their way from one QW to another. This means that QDs play the role of a sole reservoir of electrons (holes) for the QW and the OCL region adjacent to $p$-cladding ( $n$-cladding). Therefore, the density of minority carriers in these regions will be negligible. Hence, outside the QDs there will be no region in the structure where both electron and hole densities are simultaneously high. The electron density is high where the hole density is negligible, and vice versa. This strongly suppresses the parasitic components of the threshold current [the first two summands in (1)], which would otherwise give the main contribution to the temperature dependence. The spontaneous radiative recombination rate is nonvanishing solely in the QD layer.

With the parasitic recombination channels suppressed, we can expect only a slight temperature dependence of $j_{\text {th }}=j_{\mathrm{QD}}$ $\left[j_{\mathrm{QD}}\right.$ is given by the last term in (1)] caused by inhomogeneous line broadening. Defining the injection efficiency as the QD fraction of the total injection current, i.e., as $j_{\mathrm{QD}} / j$, we can write

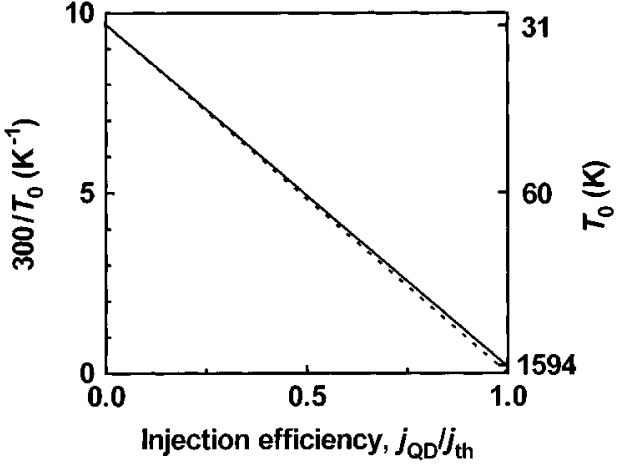

Fig. 2. Reciprocal of the characteristic temperature against injection efficiency at room temperature. The dotted line depicts $1 / T_{0}$ for the suppressed inhomogeneous line broadening $\left(T_{0}^{\mathrm{QD}}=\infty\right)$. The characteristic temperature is indicated in the right axis. The value $1594 \mathrm{~K}$ corresponds to the minimum of the $T_{0}^{Q D}$ dependence on the rms of relative QD size fluctuations (see Fig. 3).

the reciprocal of the characteristic temperature in the form (see $[12$, eq. (23)])

$$
\frac{1}{T_{0}}=\frac{j_{\mathrm{QD}}}{j_{\mathrm{th}}} \frac{1}{T_{0}^{\mathrm{QD}}}+\left(1-\frac{j_{\mathrm{QD}}}{j_{\mathrm{th}}}\right) \frac{1}{T_{0}^{\mathrm{out}}}
$$

where $T_{0}^{\mathrm{QD}}$ and $T_{0}^{\mathrm{out}}$ are defined similarly to $T_{0}$ for the threshold current components associated with the recombination in QDs $\left[j_{\mathrm{QD}}(T)\right]$ and outside the QDs [the sum of the first two terms in (1)].

The characteristic temperature $T_{0}^{\mathrm{QD}}$ is much higher than $T_{0}^{\text {out }}$. Indeed, the value of $T_{0}^{\text {out }}$ calculated for the first term in (1) is below $100 \mathrm{~K}$ at room temperature (see [12, Fig. 8]), whereas the calculated value of $T_{0}^{\mathrm{QD}}$ due to inhomogeneous line broadening is over $1000 \mathrm{~K}$ (see below). Hence, the characteristic temperature $T_{0}$ increases dramatically with increasing injection efficiency (Fig. 2). When the entire injection current is consumed in QDs, which is the case in the tunneling-injection QD laser, the dominant remaining contribution to temperature dependence is from inhomogeneous broadening and the characteristic temperature $T_{0}=T_{0}^{\mathrm{QD}}$ should be very high.

To evaluate $T_{0}$ in this limit, we first calculate $j_{\mathrm{QD}}=$ $\left(e N_{\mathrm{S}} / \tau_{\mathrm{QD}}\right)\left\langle f_{\mathbf{n}}(a) f_{\mathrm{p}}(a)\right\rangle=\left(e N_{\mathrm{S}} / \tau_{\mathrm{QD}}\right) \int f_{\mathbf{n}}(a) f_{\mathrm{p}}(a) w(a) d a$ [where $w(a)$ is the QD size distribution function], assuming that $f_{\mathrm{n}, \mathrm{p}}(a)$ are given by the Fermi-Dirac distribution function. Expanding $f_{\mathrm{n}, \mathrm{p}}(a)$ about $a=\bar{a}$, correct to third order, we obtain (5), as shown at the bottom of the page, where $\delta$ is the root mean square (rms) of the relative QD size fluctuations, $\bar{f}_{\mathrm{n}}=f_{\mathrm{n}}(\bar{a})$, and $\bar{\varepsilon}_{\mathrm{n}, \mathrm{p}}^{\prime}$ and $\bar{\varepsilon}_{\mathrm{n}, \mathrm{p}}^{\prime \prime}$ are, respectively, the first and the second derivatives of the quantized energies $\varepsilon_{\mathbf{n}, \mathbf{p}}$ with respect to the QD size taken at $a=\bar{a}$. For a Gaussian

$$
\frac{T_{0}}{T}=\frac{1-\frac{\bar{a}^{2} \delta^{2}}{2}\left(1-\bar{f}_{\mathrm{n}}\right)\left[\frac{\bar{\varepsilon}_{\mathrm{n}}^{\prime \prime}+\bar{\varepsilon}_{\mathrm{p}}^{\prime \prime}}{T}-\frac{\bar{\varepsilon}_{\mathrm{n}}^{\prime}}{T} \frac{\bar{\varepsilon}_{\mathrm{p}}^{\prime}}{T}\left(1-\bar{f}_{\mathrm{n}}\right)+\frac{\bar{\varepsilon}_{\mathrm{n}}^{2}+\bar{\varepsilon}_{\mathrm{p}}^{\prime 2}}{T^{2}}\left(2 \bar{f}_{\mathrm{n}}-1\right)\right]}{\frac{\bar{a}^{2} \delta^{2}}{2}\left(1-\bar{f}_{\mathrm{n}}\right)\left[\frac{\bar{\varepsilon}_{\mathrm{n}}^{\prime \prime}+\bar{\varepsilon}_{\mathrm{p}}^{\prime \prime}}{T}-2 \frac{\bar{\varepsilon}_{\mathrm{n}}^{\prime}}{T} \frac{\bar{\varepsilon}_{\mathrm{p}}^{\prime}}{T}\left(1-\bar{f}_{\mathrm{n}}\right)+2 \frac{\bar{\varepsilon}_{\mathrm{n}}^{\prime 2}+\bar{\varepsilon}_{\mathrm{p}}^{\prime 2}}{T^{2}}\left(2 \bar{f}_{\mathrm{n}}-1\right)\right]}
$$




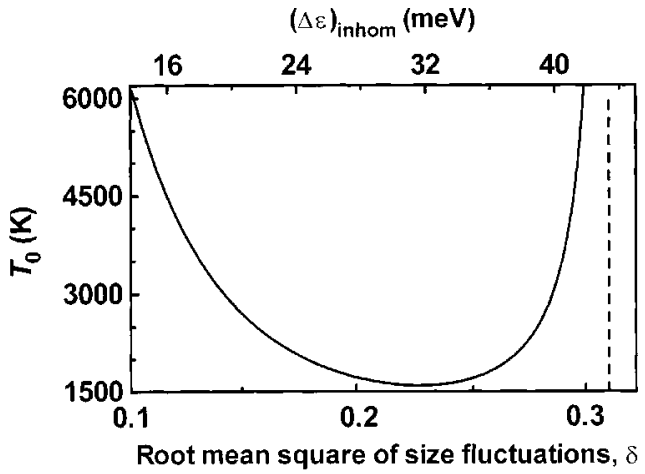

Fig. 3. Characteristic temperature against root mean square of relative QD size fluctuations (the bottom axis) and the inhomogeneous line broadening $(\Delta \varepsilon)_{\text {inlom }}=\left(\left|\bar{\varepsilon}_{\mathrm{n}}^{\prime}\right|+\left|\bar{\varepsilon}_{\mathrm{p}}^{\prime}\right|\right) \bar{a} \delta$ (the top axis). The vertical dashed line indicates

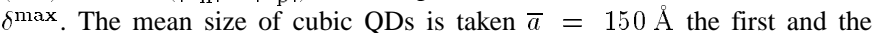
second derivatives of $\varepsilon_{\mathrm{n}, \mathrm{p}}$ with respect to the QD size are calculated using the equations for the quantized energies in a 3-D infinitely deep square well.

distribution, the full-width at half-maximum (FWHM) is $(2 \sqrt{2} \ln 2) \delta \approx 2 \delta$. In (5), the level occupancy at the lasing threshold is $\bar{f}_{\mathrm{n}}=(1 / 2)\left(1+\delta / \delta^{\max }\right)$, where $\delta^{\max }$ is the maximum tolerable QD size fluctuation, beyond which no lasing is possible (see [18, eq. (25)])

The dependence of $T_{0}=T_{0}^{\mathrm{QD}}$ on $\delta$ is nonmonotonic (Fig. 3). The decrease of $T_{0}$ at small $\delta$ is due to the increasing thermal population of the nonlasing QDs. At large QD size dispersion, when $\delta \rightarrow \delta^{\max }, \bar{f}_{\mathrm{n}}$ must approach unity to satisfy the generation condition. Full occupancy (quasi-Fermi levels $E_{\mathrm{F}} \gg k T$ ) forces temperature independence of $j_{\mathrm{QD}}$ at the price of an extremely high total current. Needless to say, large QD size dispersion is not a desired limit.

As seen from Fig. 3, the device is practically temperature insensitive over the entire range of $\delta$ with even the smallest $T_{0}$ (attained with a QD size distribution of FWHM $\approx 46 \%$ ) being over $1500 \mathrm{~K}$. Such a $T_{0}$ can be essentially considered infinite for most practical purposes. We emphasize that this dramatic improvement results solely from the suppression of recombination channels outside the active region.

It interesting to note, however, that another still "finer" effect in the proposed structure will further enhance its temperature stability. This effect stems from the resonant nature of tunneling injection which leads to an effective narrowing of the inhomogeneous linewidth. Indeed, such a mechanism of injection inherently selects the QDs of the "right" size, since it requires the confined-carrier levels to be in resonance with the lowest subband states in the QW. When this condition is met by the QDs of average size, i.e., when QDs with $a=\bar{a}$ are resonant, the number of active QDs will be maximized. Consider this situation. For QDs with $a>\bar{a}$, tunneling transitions can only be mediated by phonons. The rates of such transitions are much lower and can be safely neglected. Hence, QDs of sizes larger than the average are effectively cut off. Smaller-size QDs are also cut off, although perhaps less efficiently, because their energy levels would be pumped from higher-momentum states in the injecting QW subband. The higher the in-plane momentum of a 2-D carrier

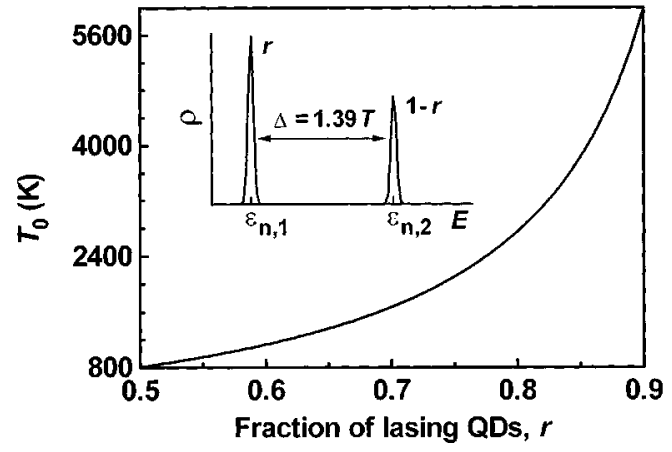

Fig. 4. Characteristic temperature against fraction of the lasing QDs. As a model, an ensemble of QDs of two characteristic sizes is considered (see the Appendix). QDs of the larger size $\left(a_{1}\right)$ are assumed to be in resonance with the QWs. $\Delta=\varepsilon_{\mathbf{n}, 2}-\varepsilon_{\mathbf{n}, 1}$ is the separation between the quantized energy levels in the QDs of two sizes. The value of $\Delta / T \approx 1.39$, at which $T_{0}$ is a minimum for $r=0.5$ (see Fig. 5), is taken. The inset shows the density of states in QDs.

in the QW, the lower is the probability of tunneling transition that results in its capture by the QD.

Selective injection means that the nonlasing QDs are not pumped either. As a result, the threshold current will decrease and the temperature stability of $j_{\text {th }}$ will be further enhanced. This is shown in Fig. 4, where $T_{0}$ is plotted against the fraction $r$ of the lasing QDs. As $r \rightarrow 1, T_{0} \rightarrow \infty$ [see also (A2)].

In this article, for the purpose of a general discussion of the advantages of tunneling-injection QD lasers, we did not intentionally focus on the specific details of the device design. Calculations for different possible structures are now in progress.

\section{CONCLUSION}

A novel approach to QD laser design has been proposed. The approach is based on tunneling injection of carriers into the QDs and offers the possibility of achieving an ultrahigh temperature stability - the key desired advantage of QD lasers. Tunneling injection suppresses recombination outside the QDs, which is the main cause for the temperature dependence of threshold current in QD lasers. The characteristic temperature $T_{0} \approx 1500 \mathrm{~K}$ has been shown to be attainable with tunneling injection. Still further enhancement of $T_{0}$ is possible owing to an inherently suppressed pumping of the nonlasing QDs.

In the proposed structure, the only remaining contribution to temperature dependence of $j_{\text {th }}$ will result from the violation of charge neutrality in QDs. The slight temperature dependence of $j_{\mathrm{QD}}$ arises from the independent variation of $f_{\mathrm{n}}(\bar{a})$ and $f_{\mathrm{p}}(\bar{a})$. Unconstrained by neutrality the occupancies of the electron and hole levels in the QD are no longer fixed by the generation condition and become temperature dependent [12]. Consideration of the neutrality-violation effect in the tunneling-injection QD lasers is beyond the scope of this article. It suffices to point out that, in a conventional QD laser, the calculated value of $T_{0}$ limited by this effect is over $1000 \mathrm{~K}$ at room temperature (see [12, Fig. 8]). Moreover, the resonant nature of tunneling injection should favor the correlation of the occupancies of any given QD by electrons and holes. In an idealized structure, we can expect that all the active QDs will remain neutral and then $T_{0}$ will be literally infinite. 


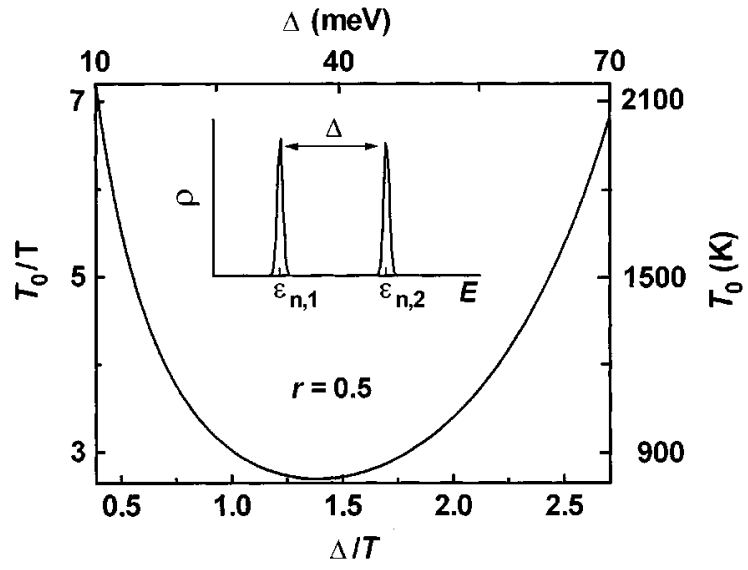

Fig. 5. Normalized characteristic temperature $T_{0} / T$ (the left axis) against normalized separation between the quantized energy levels in the QDs of two sizes $\Delta / T$ (the bottom axis). $T_{0}$ (in degrees Kelvin at room temperature) is indicated in the right axis with $\Delta$ (in $\mathrm{meV}$ ) indicated on the top axis. The inset shows the density of states in QDs. The fraction of the lasing QDs is $r=0.5$.

\section{APPENDIX \\ CHARACTERISTIC TEMPERATURE $T_{0}$ FOR A MODEL QD ENSEMBLE}

To illustrate the effect of inhomogeneous line broadening, let us consider a model ensemble of QDs of two characteristic sizes $a_{1}$ and $a_{2}$. Let us assume that QDs of the larger size $\left(a_{1}\right)$ are lasing. In a conventional QD laser, the current density component associated with the recombination in QDs is given by

$$
j_{\mathrm{QD}}=\frac{e N_{\mathrm{S}, 1}}{\tau_{\mathrm{QD}}} f_{\mathrm{n}}^{2}+\frac{e N_{\mathrm{S}, 2}}{\tau_{\mathrm{QD}}} \frac{1}{\left[\left(\frac{1}{f_{\mathrm{n}}}-1\right) \exp \left(\frac{\Delta}{T}\right)+1\right]^{2}}
$$

where

$$
\begin{array}{ll}
N_{\mathrm{S}, 1}, N_{\mathrm{S}, 2} & \text { surface densities of the QDs of two sizes; } \\
f_{\mathbf{n}} & \text { level occupancy in the QDs of the larger } \\
& \text { size; } \\
\Delta=\varepsilon_{\mathrm{n}, 2}-\varepsilon_{\mathrm{n}, 1} & \text { separation between the quantized energy } \\
& \text { levels in the QDs of two sizes (we assumed } \\
& f_{\mathbf{n}}=f_{\mathrm{p}}, \varepsilon_{\mathrm{n}, 2}-\varepsilon_{\mathbf{n}, 1}=\varepsilon_{\mathrm{p}, 2}-\varepsilon_{\mathrm{p}, 1} \text {, and } \\
& \text { the thermal equilibrium between QDs) }
\end{array}
$$

Equation (A1) illustrates the contribution to the temperature dependence of $j_{\mathrm{QD}}$ in a conventional QD laser caused by inhomogeneous broadening. It arises due to the thermal population of those QDs that are not contributing to the lasing (in this model, they are lumped into one group of smaller size $a_{2}$ ). From this effect alone, in the absence of any other mechanism for temperature dependence, the characteristic temperature would be finite (though very high)

$$
\begin{aligned}
& \frac{T_{0}}{T}=\frac{1}{1-r} \frac{\left[\left(\frac{1}{f_{\mathrm{n}}}-1\right) \exp \left(\frac{\Delta}{T}\right)+1\right]^{3}}{2 \frac{\Delta}{T}\left(\frac{1}{f_{\mathrm{n}}}-1\right) \exp \left(\frac{\Delta}{T}\right)} \\
& \left\{r f_{\mathrm{n}}^{2}+\frac{1-r}{\left[\left(\frac{1}{f_{\mathrm{n}}}-1\right) \exp \left(\frac{\Delta}{T}\right)+1\right]^{2}}\right\}
\end{aligned}
$$

where $r=N_{\mathrm{S}, 1} /\left(N_{\mathrm{S}, 1}+N_{\mathrm{S}, 2}\right)$ is the fraction of the QDs contributing to lasing.

Fig. 5 shows the characteristic temperature $T_{0}$ (normalized to $T$ ) as a universal function of $\Delta / T$. Note that $\Delta$ plays the role of inhomogeneous line broadening (contrast with Fig. 3). Like in Fig. 3, a decrease of $T_{0}$ at small $\Delta$ is caused by the increasing thermal population of the nonlasing QDs. However, the increase of $T_{0}$ at large $\Delta$ occurs here for a different reason, peculiar to the bi-modal ensemble of QDs we are considering. It is simply explained by the decreasing thermal population of the smaller-size QDs with increasing $\Delta$. In contrast to the Gaussian ensemble, this model does not show an asymptotic divergence of $T_{0}$ as in Fig. 3 , since there is no critical $\Delta$ analogous to $\delta^{\text {max }}$ and increasing $\Delta$ at a constant $r$ does not force $f_{\mathrm{n}} \rightarrow 1$.

\section{REFERENCES}

[1] Y. Arakawa and H. Sakaki, "Multidimensional quantum well laser and temperature dependence of its threshold current," Appl. Phys. Lett., vol. 40, no. 11, pp. 939-941, June 1982.

[2] N. Kirstädter, N. N. Ledentsov, M. Grundmann, D. Bimberg, V. M. Ustinov, S. S. Ruvimov, M. V. Maximov, P. S. Kop'ev, Zh. I. Alferov, U. Richter, P. Werner, U. Gösele, and J. Heydenreich, "Low threshold, large $T_{0}$ injection laser emission from (InGa)As quantum dots," Electron. Lett., vol. 30, no. 17, pp. 1416-1417, Aug. 1994.

[3] R. Mirin, A. Gossard, and J. Bowers, "Room temperature lasing from InGaAs quantum dots," Electron. Lett., vol. 32, no. 18, pp. 1732-1734, Aug. 1996.

[4] N. N. Ledentsov, M. Grundmann, F. Heinrichsdorff, D. Bimberg, V. M. Ustinov, A. E. Zhukov, M. V. Maximov, Zh. I. Alferov, and J. A. Lott, "Quantum-dot heterostructure lasers," IEEE J. Select. Topics Quantum Electron., vol. 6, pp. 439-451, May/June 2000.

[5] P. Bhattacharya, D. Klotzkin, O. Qasaimeh, W. Zhou, S. Krishna, and D. Zhu, "High-speed modulation and switching characteristics of In(Ga)As-Al(Ga)As self-organized quantum-dot lasers," IEEE $J$. Select. Topics Quantum Electron., vol. 6, pp. 426-438, May/June 2000.

[6] L. Harris, D. J. Mowbray, M. S. Skolnick, M. Hopkinson, and G. Hill, "Emission spectra and mode structure of InAs/GaAs self-organized quantum dot lasers," Appl. Phys. Lett., vol. 73, no. 7, pp. 969-971, Aug. 1998.

[7] P. M. Smowton, E. J. Johnston, S. V. Dewar, P. J. Hulyer, H. D. Summers, A. Patane, A. Polimeni, and M. Henini, "Spectral analysis of InGaAs/GaAs quantum-dot lasers," Appl. Phys. Lett., vol. 75, no. 15, pp. 2169-2171, Oct. 1999.

[8] D. L. Huffaker, G. Park, Z. Zou, O. B. Shchekin, and D. G. Deppe, "Continuous-wave low-threshold performance of $1.3-\mu \mathrm{m}$ InGaAs-GaAs quantum-dot lasers," IEEE J. Select. Topics Quantum Electron., vol. 6, pp. 452-461, May/June 2000

[9] M. Sugawara, K. Mukai, Y. Nakata, K. Otsubo, and H. Ishilkawa, "Performance and physics of quantum-dot lasers with Self-assembled columnar-shaped and 1.3- $\mu \mathrm{m}$ emitting InGaAs quantum dots," IEEE $J$. Select. Topics Quantum Electron., vol. 6, pp. 462-474, May/June 2000.

[10] L. F. Lester, A. Stintz, H. Li, T. C. Newell, E. A. Pease, B. A. Fuchs, and K. J. Malloy, "Optical characteristics of 1.24- $\mu \mathrm{m}$ InAs quantum-dot laser diodes," IEEE Photon. Technol. Lett., vol. 11, pp. 931-933, Aug. 1999.

[11] J. K. Kim, R. L. Naone, and L. A. Coldren, "Lateral carrier confinement in miniature lasers using quantum dots," IEEE J. Select. Topics Quantum Electron., vol. 6, pp. 504-510, May/June 2000.

[12] L. V. Asryan and R. A. Suris, "Temperature dependence of the threshold current density of a quantum dot laser," IEEE J. Quantum Electron., vol. 34, pp. 841-850, May 1998.

[13] _ "Inhomogeneous line broadening and the threshold current density of a semiconductor quantum dot laser," Semicond. Sci. Technol., vol. 11, no. 4, pp. 554-567, Apr. 1996.

[14] _ "Charge neutrality violation in quantum dot lasers," IEEE J. Select. Topics Quantum Electron., vol. 3, pp. 148-157, Apr. 1997.

[15] H. Yoon, A. Guitierrez-Aitken, R. Jambunathan, J. Singh, and P. Bhattacharya, "A 'cold' InP-based tunneling injection laser with greatly reduced auger recombination and temperature dependence," IEEE Photon. Technol. Lett., vol. 7, pp. 974-976, 1992. 
[16] P. Bhattacharya, J. Singh, H. Yoon, X. Zhang, A. Gutierrez-Aitken, and Y. Lam, "Tunneling injection lasers: A new class of lasers with reduced hot carrier effects," IEEE J. Quantum Electron., vol. 32, pp. 1620-1629, Sept. 1996.

[17] D. Klotzkin and P. Bhattacharya, "Temperature dependence of dynamic and DC characteristics of quantum-well and quantum-dot lasers: A comparative study," IEEE J. Lightwave Technol., vol. 17, pp. 1634-1642, Sept. 1999.

[18] L. V. Asryan and R. A. Suris, "Longitudinal spatial hole burning in a quantum-dot laser," IEEE J. Quantum Electron., vol. 36, pp. 1151-1160, Oct. 2000.

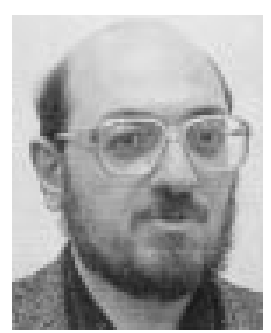

Levon V. Asryan was born in Talin, Armenia, in 1963. He received the M.Sc. degree in radiophysics and electronics from the Yerevan State University, Armenia, in 1985, and the Ph.D. degree in physics and mathematics from the Ioffe Physico-Technical Institute, Saint Petersburg, Russia, in 1988.

Since 1992, he has been a member of Technical Staff at the Ioffe Institute (Senior Member since 1999). He is currently a Senior Visiting Scientist at the State University of New York at Stony Brook, Stony Brook, NY. His research interests include the physics and performance characteristics of semiconductor lasers. He has developed, in association with Prof. R. Suris, a theory of threshold characteristics of quantum dot lasers, and has produced a series of key publications on the theory of quantum-dot lasers. From 1995 to 1998, he collaborated with the Nortel Networks (Canada) on the simulation of threshold and high-power characteristics of multiple quantum-well lasers.

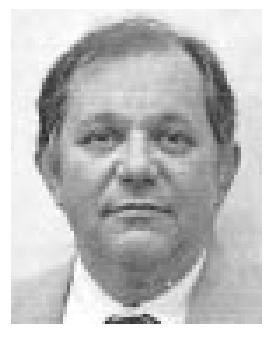

Serge Luryi (M'81-SM'85-F'90) received the $\mathrm{Ph} . \mathrm{D}$. degree in physics from the University of Toronto, ON, Canada, in 1978. His doctoral thesis was devoted to quantum mechanics of intermolecular interactions in solid hydrogen.

In 1980, he joined Bell Laboratories, where he became interested in the physics and technology of semiconductor devices. In 1994, he joined the State University of New York at Stony Brook, Stony Brook, NY, where he is currently a Leading Professor and Chair of Electrical and Computer Engineering. His other major activity is as Director of the Center for Advanced Technology in Sensor Systems. Among his research activities, he leads a large interdisciplinary research program devoted to the development of novel fluorescent sensors for DNA sequencing. He has published over 160 scientific papers, holds 30 patents, and has edited four technical books. In 1995, he organized and served as the Director of an advanced research workshop (NATO ARW, Ile de Bendor, France) on the "Future Trends in Microelectronics: Reflections on the Road to Nanotechnology." A second workshop in this series, "FTM: Off the Beaten Path" took place in June 1998, and the third is scheduled for June 2001

Dr. Luryi served as the Editor of IEEE TRANSACTIONS ON ELECTRON DEVICES during 1986-1990. He was elected Fellow of the IEEE for contributions in the field of heterojunction devices in 1989, received the Distinguished Member of Technical Staff Award from Bell Laboratories in 1990, and was elected Fellow of the American Physical Society for his theory of electron transport in low-dimensional systems and invention of novel electron devices in 1993. 